\title{
A criação da Liga Acadêmica Interprofissional em Atenção à Saúde durante a pandemia e o seu impacto na formação de universitários
}

\author{
The creation of the Interprofessional Academic League in Health Care during the \\ pandemic and its impact on the education of undergraduate students
}

\section{La creación de la Liga Académica Interprofesional en Salud durante la pandemia y su impacto en la formación de estudiantes universitarios}

\begin{abstract}
Gilberto da Cruz Leal ${ }^{1 *}$, Andréa Gracindo da Silva ${ }^{1}$, Laura Lima Costa ${ }^{1}$, Bruna Marques Maran ${ }^{1}$, Letícia Detore Develey ${ }^{1}$, Sthefany Santos Araujoㄹ, Isabella Arantes Margarido', Juliana Aparecida de Brito Baptista ${ }^{1}$, Carlos Eduardo Capelini Eli Lopes ${ }^{1}$, Regina Yoneko Dakuzaku Carretta1.
\end{abstract}

\section{RESUMO}

Objetivo: Relatar a experiência de graduandos da área da saúde de uma universidade pública do interior do estado de São Paulo, integrantes de uma Liga acadêmica interprofissional, fundada no ano de 2020, durante a pandemia do coronavírus. Relato de experiência: A presente Liga é composta por estudantes de nove cursos de graduação de Educação Física, Enfermagem, Farmácia, Fisioterapia, Fonoaudiologia, Informática Biomédica, Medicina, Nutrição e Metabolismo, Odontologia e Terapia Ocupacional. É um espaço para o aprendizado que vai além dos assuntos usualmente discutidos em sala de aula que, na maioria das vezes, são focados em assuntos uniprofissionais. A implementação das práticas nesse primeiro ano mostrou-se desafiadora, principalmente porque exigiu dos estudantes uma considerável desenvoltura para a implementação de todas as atividades de forma remota. Grande parte dos integrantes da liga não chegaram a se conhecer pessoalmente e/ou não vivenciaram aulas presenciais. Considerações finais: Os recursos utilizados (TICs) levaram a maior integração entre os membros da entidade acadêmica, bem como o alcance de um público mais amplo e diversificado.

Palavras-chave: Educação interprofissional, Educação em saúde, Tecnologia da informação.

\begin{abstract}
Objective: Reporting the experience of undergraduate health students at a public university in the interior of the State of São Paulo, members of an interprofessional academic League, founded in 2020, during the coronavirus pandemic. Experience report: This League is composed of students from nine undergraduate courses on Physical Education, Nursing, Pharmacy, Physiotherapy, Speech Language Therapy, Biomedical Informatics, Medicine, Nutrition and Metabolism, Dentistry and Occupational Therapy. It is a space for learning that goes beyond the subjects usually discussed in the classroom, which, in most cases, are focused on uniprofessional matters. The implementation of practices in this first year proved to be challenging, mainly because it required a considerable resourcefulness from the students to implement all activities in a remote way. Most of the members of the league didn't get to know each other and/or did not experience in person classes. Final considerations: The used resources (ICTs) leaded to a greater integration among the members of the academic entity, as well as the reaching of a wider and more diversified audience.
\end{abstract}

Keywords: Interprofessional education, Health education, Information technology.

1 Universidade de São Paulo (USP), Ribeirão Preto - SP. *E-mail: gillbertoleal@usp.br 


\section{RESUMEN}

Objetivo: Informar sobre la experiencia de los estudiantes de pregrado del área de salud de uma universidad pública del interior del Estado de São Paulo, integrantes de una Liga académica interprofesional, fundada en 2020, durante la pandemia de coronavirus. Informe de experiencia: Esta Liga está compuesta por estudiantes de nueve cursos de pregrado de Educación Física, Enfermería, Farmacia, Fisioterapia, Terapia del lenguaje, Informática Biomédica, Medicina, Nutrición y Metabolismo, Odontología y Terapia Ocupacional. Es un espacio de aprendizaje que va más allá de los temas tratados en la clase, que en la mayoría de los casos están enfocados en asuntos uniprofesional. La implementación de prácticas en este primer año resultó ser un desafío, principalmente porque requirió de los estudiantes un considerable ingenio para implementar todas las actividades de forma remota. La mayoría de los miembros de la liga no se conocieron y/o no tuvieron clases presenciales. Consideraciones finales: Los recursos utilizados (TICs) levaran a una mayor integración entre los miembros de la entidad académica, así como el alcance de un público más amplio y diversificado.

Palabras clave: Educación interprofesional, Educación en salud, Tecnología de la información.

\section{INTRODUÇÃO}

As ligas acadêmicas são entidades autônomas, sem fins lucrativos, que possuem como finalidade aprofundar o conhecimento dos envolvidos em vários temas, específicos ou não, pautados na inerência entre o ensino, a extensão e a pesquisa (SILVA SA e FLORES O, 2015; CAVALCANTE ASP, et al., 2018). Por estarem vinculadas às universidades, configuram-se em atividades extracurriculares. Ligas podem ser compostas por estudantes de um único curso ou de vários cursos; neste último caso, geralmente as ligas possuem enfoque na interprofissionalidade. Além disso, são constituídas por docentes tutores, que são os responsáveis por administrar e apresentar os assuntos (SILVA JVS, et al., 2021).

Além de aprofundar os conteúdos e complementar o ensino, as ligas proporcionam interação entre os membros; a possibilidade da aprendizagem prática; e a aproximação entre alunos, a instituição e a comunidade, através da contribuição em pesquisas, assistências e trocas de informações (SILVA JVS, et al, 2021). Dessa forma, é notável que as ligas proporcionam maior experiência profissional e crescimento pessoal aos estudantes, resultando em melhores oportunidades de trabalho e execução das funções atribuídas (CAVALCANTE ASP, et al, 2018).

Os motivos para participar de uma Liga Acadêmica são distintos, mas se assemelham entre si perante a visão de alunos e professores (YANG GY, et al., 2019). O primeiro motivo são as lacunas curriculares apresentadas ao longo da graduação: áreas do conhecimento não abordadas e/ou não aprofundadas dão espaço à criação das ligas e, consequentemente, fomentam a participação dos estudantes nessas entidades (SILVA JVS, et al., 2021). O segundo é a possibilidade dos alunos compartilharem saberes científicos sobre o tema da Liga e desenvolverem ações de extensão, aproximando-os dos professores e da comunidade. Já o terceiro se manifesta no desenvolvimento de competências e habilidades, tais como trabalho em equipe, liderança, resolução de problemas, planejamento e empreendedorismo, muito importantes para o futuro dos estudantes (YANG, et al., 2019; SILVA JVS, et al., 2021). Em suma, percebe-se a importância do protagonismo dos estudantes (ARAÚJO CRC, et al., 2018; CAVALCANTE ASP, et al., 2021).

A educação interprofissional nos últimos anos tem ganhado espaço entre os estudantes e educadores, e tem sido constantemente debatida nos contextos educacionais e de saúde. É de fundamental importância que a interprofissionalidade seja implantada nos cursos da área da saúde para que políticas públicas possam ser repensadas e reestruturadas, visando o bem comum (REEVES S, 2016; BAQUIÃO APSS, et al., 2020). Desta maneira o aluno, futuro profissional, poderá de forma colaborativa e dentro de suas competências aprender com o outro, acerca do trabalho do outro. Por conseguinte, os usuários dos serviços de saúde poderão receber um atendimento mais humanizado, com maior qualidade e com menores chances de erros ou fatalidades (BROCK D, et al., 2013; COSTA MV, 2016). 
A pandemia da COVID-19 representou um grande desafio para as instituições de ensino e para os estudantes de forma geral, principalmente pelo adiamento das atividades práticas e pela migração para 0 ensino remoto. Por conta disso, o Ministério da Educação (2020) autorizou, em caráter excepcional e emergencial, a substituição das aulas presenciais por remotas, por meio das Tecnologias de Informação e Comunicação (TICs). Apropriando-se desses recursos, os estudantes encontraram um caminho para a continuidade (ou início) de práticas extracurriculares, como as prestadas por ligas acadêmicas.

A importância do presente relato é demonstrar a efetividade e os ganhos oriundos de atividades extracurriculares na formação de universitários, mais especificamente dos provenientes da participação de uma entidade estudantil de caráter interprofissional. O objetivo do estudo foi relatar a experiência de graduandos da área da saúde de uma universidade pública do interior do estado de São Paulo, integrantes de uma Liga acadêmica interprofissional, fundada no ano de 2020, durante a pandemia do coronavírus.

\section{RELATO DE EXPERIÊNCIA}

A Liga Acadêmica Interprofissional em Atenção à Saúde (LIAS) foi idealizada por três alunos do curso de fonoaudiologia da instituição de ensino. Tais estudantes eram participantes ativos em eventos, fóruns, congressos e afins, das mais diversas áreas, e sentiram a necessidade de criar uma entidade que se articulasse de uma forma mais direta com as diversas carreiras acadêmicas, e em especial com as da área da saúde. Dois desses idealizadores fizeram parte do Programa de Educação pelo Trabalho para a Saúde (PET-Saúde), um programa dos Ministérios da Saúde e da Educação (MINISTÉRIO DA SAÚDE, 2010). Na versão de que os fundadores participaram - entre os anos de 2019 e 2021 - o tema central foi a interprofissionalidade. Diante do aprendizado adquirido, os alunos perceberam que era importante levar o conhecimento da prática interprofissional aos diversos estudantes da instituição de ensino, uma vez que poucos foram agraciados com o PET-Saúde.

Em setembro de 2020 os fundadores Andréa Gracindo e Gilberto Leal, juntamente com a tutora Regina Carretta, criaram o estatuto da LIAS. Após algumas reuniões definiram os princípios e finalidades da Liga, tanto no ensino quanto na pesquisa e na extensão. Um mês depois o processo seletivo foi iniciado e contou com ampla divulgação nas redes sociais e nos veículos de imprensa da universidade. O convite foi estendido a estudantes dos cursos da saúde de todo o campus. Após dois meses de divulgação (dezembro), as entrevistas com os candidatos foram realizadas, contando com a participação de 40 inscritos. Por se tratar de um período pandêmico, todo processo ocorreu de forma online, com apoio da plataforma Google Meet. Cada candidato escolheu o melhor horário para a sua entrevista, quando foram abordados temas como motivações, possíveis contribuições e expectativas de cada um.

Do total de inscritos, 21 estudantes foram selecionados. A LIAS foi fundada com representantes de nove cursos, sendo eles: Educação Física, Enfermagem, Farmácia, Fisioterapia, Fonoaudiologia, Informática Biomédica, Medicina, Nutrição e Metabolismo, Odontologia e Terapia Ocupacional. Aos estudantes que não foram selecionados para compor a primeira gestão foi proporcionada a política de portas-abertas, que possibilitou a participação destes na categoria "ligantes", isto é, indivíduos que não fazem parte da gestão, mas que vivenciam todo o processo de criação e organização de eventos e que recebem certificados pela participação.

Aos membros, todavia, foram designadas atividades, segmentadas em departamentos. Os idealizadores e a tutora da LIAS fizeram sugestões de cargos a partir das informações das entrevistas, levando em conta as habilidades e experiências dos estudantes. Entretanto, o membro poderia optar por outra área. Inicialmente, a liga contou com os seguintes departamentos: presidência, secretaria, financeiro, comunicação/divulgação, científico e eventos. Cada departamento, exceto o da presidência, tem um(a) diretor(a) e seus respectivos coordenadores.

Após o início das atividades, os membros decidiram que era viável a criação de uma equipe que atuasse diretamente com a proposta de Cultura e Extensão, dadas as demandas e potencialidades de atuação nesse campo. A finalidade era estabelecer parcerias com entidades que atuassem em prol de projetos humanitários e de interesse da sociedade (GALVÃO P e ARAÚJO CMM, 2018). 
As organizações que realizaram parceria com a LIAS possuíam diversas finalidades, como por exemplo, apoio à mulher e à criança, dentre outros. As parcerias acontecem de forma remota; a Liga divulga as necessidades das entidades por meio de publicações nas redes sociais. Tais divulgações são de âmbito arrecadatório (alimentos e roupas) e de promoção de eventos sociais. Ao todo, a LIAS fez parcerias com 5 entidades na primeira gestão.

A LIAS promoveu eventos científicos tanto internos quanto externos. Os internos visaram o nivelamento dos membros acerca da interprofissionalidade, além do compartilhamento de experiências. Os externos, por outro lado, tinham como público-alvo universitários de cursos da saúde, profissionais, ou interessados. As temáticas dos eventos foram estabelecidas através das demandas dos próprios membros, das datas comemorativas ou das caixas de sugestões disponíveis em nossas redes sociais.

Um fato importante a se destacar é que os eventos foram realizados exclusivamente pelos membros da Liga ou por meio de parcerias com outras entidades acadêmicas. Foram abordados temas como "A saúde dos estudantes durante o isolamento social", a "Saúde da mulher: vaginismo" e a "Visibilidade LGBTQIA+". Todos os eventos foram realizados de forma remota e as palestras proferidas por pesquisadores, mestres e doutores, bem como por graduandos de diferentes áreas.

Devido ao interesse por temas que retratam a Interprofissionalidade, a sua importância, os desafios, e a atuação nos ambientes universitários e profissionais, surgiu o desejo pela realização de um simpósio. Após meses de planejamento, ocorreu o I Simpósio Interprofissional em Saúde da instituição. O evento foi realizado remotamente entre os dias 18 e 20 de agosto de 2021, e transmitido pelo YouTube. O público-alvo foram graduandos e pós-graduandos de cursos da saúde, profissionais, ou interessados, de todo país. Para a inscrição foi criado um formulário online, disponibilizado com o material de divulgação. Todos os inscritos receberam certificados de participação.

No primeiro dia de simpósio contamos com a presença de experts que apresentaram e debateram os temas "A Educação Interprofissional e a Prática Colaborativa" e "Perspectivas e Limites da Educação Interprofissional: como estamos nos relacionando" em uma mesa redonda, com a participação de um moderador. Na segunda noite, ocorreram palestras ministradas por mestres e doutores com os temas: "Interprofissionalidade no ensino da graduação em saúde: desafios e potencialidades vivenciadas pelos estudantes", "Interprofissionalidade durante a graduação em saúde, experiências de aprendizado e pesquisas no âmbito do PET Saúde IP da Universidade de São Paulo e da Secretaria Municipal de Saúde de Ribeirão Preto, 2019 a 2021", e "Formação para a Interprofissionalidade: duas experiências, um propósito".

As apresentações retrataram a atuação dos estudantes frente à interprofissionalidade, o desenvolvimento do olhar sobre o paciente, a necessidade do aperfeiçoamento da escuta ativa e da comunicação não violenta, e as futuras expectativas para a implementação deste tipo de prática entre os cursos de graduação e na atuação profissional. De forma comum, os temas destacaram a importância da integração e interação entre estudantes para um trabalho em equipe mais efetivo (FOX L, et al., 2018; LIMA AWS, et al., 2020). O último dia contou com a apresentação oral de trabalhos e premiação dos melhores classificados. Os resumos previamente submetidos foram avaliados e selecionados por mestres e doutores, sendo os aceitos enviados para publicação em formato de anais do evento, em uma revista universitária.

\section{DISCUSSÃO}

A participação na LIAS tem proporcionado aos seus membros um olhar rico e diferenciado acerca da interprofissionalidade; é constante o relato de estudantes que se mostram surpresos frente ao conhecimento das possibilidades de atuação do outro, extrapolando significativamente o senso comum.

Sabe-se que a interprofissionalidade é entendida como a articulação entre duas ou mais profissões ou profissionais, sendo uma ferramenta educacional que "surgiu com o propósito de melhorar a qualidade da atenção à saúde ao paciente a partir do efetivo trabalho em equipe, no ponto de vista da prática colaborativa" (CÔRREA JMC, et al., 2018). O contato entre estudantes e profissionais de outras áreas desde a formação acadêmica incentiva o trabalho harmônico em equipe e ajuda a reduzir os conflitos profissionais que eventualmente possam surgir. 
Outro ponto a se destacar é a importância da LIAS enquanto geradora de conhecimento e informação, na medida em que alguns estudantes, por exemplo, não sabiam que educadores físicos eram profissionais da saúde. Nesse sentido, a Liga permitiu um espaço de aprendizado que vai além dos discutidos dentro da sala de aula que, na maioria das vezes, é focado no uniprofissional. Através de tais espaços, os alunos puderam aprender mais sobre o que o outro faz e quais as fronteiras entre cada profissão. Dentro deste cenário de integração e amplitude de conhecimentos, os estudantes tiveram a possibilidade de pensar em parcerias para elaboração de projetos integrando profissões distintas outrora não imaginados.

Eventos, conferências e simpósios online se multiplicaram de maneira significativa com o advento do isolamento social imposto pela pandemia. Por conta disso, houve um boom de lives e videoconferências nas redes (SILVA WBH, et al., 2020). Tais ferramentas trouxeram impactos potentes ao permitir o acesso amplo e gratuito a informações de confiança difundidas entre pessoas de localizações geográficas longínquas. Como exemplo disso, destaca-se o simpósio mencionado, que incluiu participantes de nove estados brasileiros (Amazonas, Bahia, Espírito Santo, Goiás, Minas Gerais, Paraná, Rio de Janeiro, Rio Grande do Sul e São Paulo) e também do Distrito Federal. O evento contou com 302 visualizações em sua transmissão ao vivo pelo Youtube.

Todos os eventos promovidos pela liga foram gravados com o consentimento dos palestrantes e disponibilizados no YouTube. Ao todo, contamos com 1186 visualizações em nosso canal oficial. Os eventos ficarão disponíveis na plataforma por um tempo indeterminado. Tal fato possibilitou levar informação para a população por mais tempo, podendo alcançar mais pessoas e, consequentemente levando o trabalho e as oportunidades que a Liga tem a oferecer ao seu público.

A partir do exposto, fica claro que mesmo com o fim da pandemia, a realização de eventos online e/ou híbridos é um caminho que tem muito a oferecer, principalmente porque permite a aproximação de estudantes e profissionais das mais diversas regiões brasileiras (SANTARATO N, et al., 2021).

Por outro lado, é importante ressaltar que a produção de eventos online é marcada por dificuldades como a falta de conexão com a internet, as interrupções eventuais ou ainda a lentidão das transmissões (SANTARATO N, et al., 2021). Além disso há também outros desafios como a falta da interação presencial (que resulta em relacionamentos superficiais entre os participantes), a perda do networking (que se dá, principalmente, no coffee-break) e o risco de dispersão da audiência frente às telas.

Ressalta-se que a participação em uma entidade acadêmica não é obrigatória, embora represente muitos benefícios para os estudantes. A LIAS acredita ter criado um espaço que permite troca de experiências riquíssimas, que muito têm a agregar. Santos LC, et al. (2018) destacam que em muitas situações a questão curricular e/ou institucional limita a integração entre os cursos de graduação, o que ficou evidente em muitos momentos da graduação, principalmente para alunos em períodos de conclusão acadêmica. Nesse sentido, vemos a Liga como agente de transformação.

A implementação das práticas nesse primeiro ano mostrou-se desafiadora porque exigiu dos estudantes uma considerável desenvoltura para a implementação de todas as atividades de forma remota. Grande parte dos integrantes da liga não chegaram a se conhecer pessoalmente ou não vivenciaram aulas presenciais. No entanto, considera-se que os recursos utilizados (TICs) levaram a uma maior integração entre os membros da entidade acadêmica, bem como o alcance de um público mais amplo e diversificado que por diversos motivos não vivencia a rotina da instituição de ensino. Além disso, o presente relato pode contribuir como orientação para estudantes de todo país, na medida em que são poucas as entidades acadêmicas formadas especificamente no período pandêmico, e consequentemente, poucos são os relatos existentes na literatura.

Por fim, reconhece-se que a LIAS propiciou aos seus membros um maior aprendizado acerca da interprofissionalidade, bem como uma maior aproximação com os diversos agentes sociais, além de viabilizar o aperfeiçoamento de habilidades relacionadas ao desenvolvimento, planejamento e organização de eventos, aulas e simpósio com temáticas relacionadas à saúde física, mental e social, com foco na educação interprofissional em saúde. 


\section{REFERÊNCIAS}

1. ARAÚJO CRC, et al. Contribuição das ligas acadêmicas para o processo de ensino-aprendizagem na graduação em enfermagem. Revista tendências da enfermagem profissional, 2018; 10(3): 3-8.

2. BAQUIÃO APSS, et al. Educação interprofissional em saúde: revisão integrativa da literatura brasileira (2008-2018). Revista Psicologia e Saúde, 2020; 12(4): 125-139.

3. BROCK D, et al. Interprofessional education in team communication: working together to improve patient safety. Postgraduate Medical Journal, 2013; 89(1057): 642-651.

4. CAVALCANTE ASP, et al. As ligas acadêmicas na área da saúde: lacunas do conhecimento na produção científica brasileira. Revista Brasileira de Educação Médica, 2018; 42(1): 199-2016

5. CAVALCANTE ASP, et al. Em busca da definição contemporânea de "ligas acadêmicas" baseada na experiência das ciências da saúde. Revista Interface (Botucatu), 2021; 25: e190857.

6. CÔRREA JMC, et al. Inserção da interprofissionalidade no âmbito da saúde. Revista Eixos Tech, 2018; 5(1): e197.

7. COSTA MV. A educação interprofissional no contexto brasileiro: algumas reflexões. Revista Interface - Comunicação, Saúde e Educação, 2016; 20(56): 197-198.

8. FOX L, et al. Teaching interprofessional teamwork skills to health professional students: a scoping review. Journal of Interprofessional Care, 2018; 32(2): 127-135.

9. GALVÃO P, ARAÚJO CMM. ONGs no Brasil: contextualização histórica do cenário para atuação em psicologia escolar. Psicologia em Revista, 2018; 24(1): 101-123.

10. LIMA AWS, et al. Perception and manifestation of collaborative competencies among undergraduate health students. Revista Latino-Americana de Enfermagem, 2020; 28: e3240.

11. MINISTÉRIO DA EDUCAÇÃO. Portaria nº 343, de 17 de março de 2020. Dispõe sobre a substituição das aulas presenciais por aulas em meios digitais enquanto durar a situação de pandemia do Novo Coronavírus - COVID-19, 2020. Disponível em: https://www.in.gov.br/en/web/dou/-/portaria-n-343-de-17-de-marco-de-2020-248564376. Acessado em: 13 de novembro de 2021.

12. MINISTÉRIO DA SAÚDE. Portaria Interministerial nำ421, de 3 de março de 2010. Institui o Programa de Educação pelo Trabalho para a Saúde (PET Saúde) e dá outras providências, 2010. Disponível em: https://bvsms.saude.gov.br/bvs/saudelegis/gm/2010/pri0421_03_03_2010.html. Acessado em: 23 de outubro de 2021.

13. REEVES S. Porque precisamos de educação interprofissional para um cuidado efetivo e seguro. Revista Interface Comunicação, Saúde e Educação, 2016; 20(56): 185-196.

14. SANTARATO N, et al. Atuação de uma liga acadêmica de cuidados à saúde da mulher durante o primeiro ano da pandemia da COVID-19. Revista Eletrônica Acervo Saúde, 2021; 13(9): e8768.

15. SANTOS LC, et al. A educação interprofissional na graduação de medicina e enfermagem em prática na atenção primária à saúde: a perspectiva dos estudantes. Revista Interface - Comunicação, Saúde e Educação, 2018; 22(supl.2): 1601:1611.

16. SILVA SA, FLORES O. Ligas acadêmicas no processo de formação dos estudantes. Revista Brasileira de Educação Médica, 2015; 39(3): 410-417.

17. SILVA JVS, et al. Liga Acadêmica interdisciplinar de Saúde Mental: ampliando a formação e as práticas no campo da atenção psicossocial. Medicina (Ribeirão Preto), 2021; 54(2): e-174130.

18. SILVA WBH, et al. Reinvenção das ligas acadêmicas em período de pandemia e interrupção das aulas presenciais. Global Academic Nursing Journal, 2020; 1(3): e51.

19. YANG GY, et al. Liga de Anatomia Aplicada (LAA): as Múltiplas Perspectivas sobre Participar de uma Liga Acadêmica. Revista Brasileira de Educação Médica, 2019; 43(1): 80-86. 